\title{
Compatibility of the new DAMA/NaI data on an annual modulation effect in WIMP direct search with a relic neutralino in supergravity schemes
}

\author{
A. Bottino, ${ }^{1, *}$ F. Donato, ${ }^{1, \dagger}$ N. Fornengo, ${ }^{1, \dagger}$ and S. Scopel ${ }^{2, \S}$ \\ ${ }^{1}$ Dipartimento di Fisica Teorica, Università di Torino \\ and INFN, Sezione di Torino, Via P. Giuria 1, 10125 Torino, Italy \\ ${ }^{2}$ Instituto de Física Nuclear y Altas Energías, Facultad de Ciencias, Universidad de Zaragoza, \\ Plaza de San Francisco s/n, 50009 Zaragoza, Spain \\ (Received 1 September 1998; published 30 March 1999)
}

\begin{abstract}
Recent results of the DAMA/NaI experiment for weakly interacting massive particle direct detection point to a possible annual modulation effect in the detection rate. We show that these results, when interpreted in terms of a relic neutralino, are compatible with supergravity models. Together with the universal supergravity (SUGRA) scheme, we also consider SUGRA models where the unification condition in the Higgs mass parameters at a grand unified theory scale is relaxed. [S0556-2821(99)03205-1]

PACS number(s): 12.60.Jv, 11.30.Pb, 14.80.Ly, 95.35.+d
\end{abstract}

\section{INTRODUCTION}

The new DAMA/NaI data (running period No. 2) [1] provide a further indication of a possible annual modulation effect in the rate for weakly interacting massive particle (WIMP) direct detection, already singled out by the same Collaboration using former data (running period No. 1) [2]. In Ref. [3] we interpret the total sample of new and former data in terms of a relic neutralino in the framework of the minimal supersymmetric extension of the standard model (MSSM) [4], by extending the analysis that we performed previously [5] about the DAMA/NaI results of the running period No. 1. The MSSM scheme represents a very versatile approach for discussing supersymmetric phenomenology at the electroweak (EW) scale, and does not bear on too strong theoretical assumptions at higher energies. In Ref. [3], using the MSSM, we prove that the annual modulation data are quite compatible with a relic neutralino which may make up the major part of the dark matter in the Universe and that some of the most relevant supersymmetric properties are explorable at accelerators in the near future.

In the present paper we show that the supersymmetric features, implied by the DAMA/NaI modulation data, are also compatible with more ambitious supersymmetry schemes, where the previous phenomenological model is implemented in a supergravity (SUGRA) framework, especially if the unification conditions, which are frequently imposed at the grand unified theory (GUT) scale, are appropriately relaxed [6].

\section{SUGRA MODELS}

We recall here that the essential elements of SUGRA models $[7,8]$ are a Yang-Mills Lagrangian, the superpoten-

\footnotetext{
*Email address: bottino@to.infn.it

†Email address: donato@to.infn.it

*Email address: fornengo@to.infn.it

${ }^{\S}$ Email address: scopel@ posta.unizar.es
}

tial, which contains all the Yukawa interactions between the standard and supersymmetric fields, and the soft-breaking Lagrangian, which models the breaking of supersymmetry. Here we only recall the soft supersymmetry breaking terms

$$
\begin{aligned}
-\mathcal{L}_{\text {soft }}= & \sum_{i} m_{i}^{2}\left|\phi_{i}\right|^{2}+\left\{\left[A_{a b}^{l} h_{a b}^{l} \widetilde{L}_{a} H_{1} \widetilde{R}_{b}\right.\right. \\
& \left.+A_{a b}^{d} h_{a b}^{d} \widetilde{Q}_{a} H_{1} \widetilde{D}_{b}+A_{a b}^{u} h_{a b}^{u} \widetilde{Q}_{a} H_{2} \widetilde{U}_{b}+\text { H.c. }\right] \\
& \left.-B \mu H_{1} H_{2}+\text { H.c. }\right\}+\sum_{i} M_{i}\left(\lambda_{i} \lambda_{i}+\bar{\lambda}_{i} \bar{\lambda}_{i}\right)
\end{aligned}
$$

where the $\phi_{i}$ are the scalar fields, the $\lambda_{i}$ are the gaugino fields, $H_{1}$ and $H_{2}$ are the two Higgs fields, $\widetilde{Q}$ and $\widetilde{L}$ are the doublet squark and slepton fields, respectively, and $\widetilde{U}, \widetilde{D}$, and $\widetilde{R}$ denote the SU(2)-singlet fields for the up-squarks, down-squarks, and sleptons. In Eq. (1), $m_{i}$ and $M_{i}$ are the mass parameters of the scalar and gaugino fields, respectively, and $A$ and $B$ denote trilinear and bilinear supersymmetry breaking parameters, respectively. The Yukawa interactions are described by the parameters $h$, which are related to the masses of the standard fermions by the usual expressions, e.g. $m_{t}=h^{t} v_{2}$ and $m_{b}=h^{b} v_{1}$, where $v_{i}=\left\langle H_{i}\right\rangle$ are the vacuum expectation values (VEV's) of the two Higgs fields.

It is worth recalling that one attractive feature of the model is the connection between soft supersymmetry breaking and electro-weak symmetry breaking (EWSB), which would then be induced radiatively. It is customary to implement the supergravity framework with some restrictive assumptions about unification at grand unification scale $M_{\mathrm{GUT}}$ : (i) unification of the gaugino masses $M_{i}\left(M_{\mathrm{GUT}}\right) \equiv m_{1 / 2}$; (ii) universality of the scalar masses with a common mass denoted by $m_{0}, m_{i}\left(M_{\mathrm{GUT}}\right) \equiv m_{0}$; (iii) universality of the trilinear scalar couplings $A^{l}\left(M_{\mathrm{GUT}}\right)=A^{d}\left(M_{\mathrm{GUT}}\right)=A^{u}\left(M_{\mathrm{GUT}}\right)$ $\equiv A_{0} m_{0}$.

As extensively discussed in Ref. [9], these conditions have strong consequences for low-energy supersymmetry phenomenology, and in particular for the properties of the 
neutralino, which is defined as the lowest-mass linear superposition of the two neutral gauginos $(\tilde{\gamma}$ and $\widetilde{Z})$ and the two neutral Higgsinos $\left(\widetilde{H}_{1}\right.$ and $\left.\widetilde{H}_{2}\right)$ :

$$
\chi=a_{1} \tilde{\gamma}+a_{2} \widetilde{Z}+a_{3} \widetilde{H}_{1}+a_{4} \widetilde{H}_{2} .
$$

Different neutralino compositions are classified in terms of the parameter $P \equiv a_{1}^{2}+a_{2}^{2}$ : gaugino-like when $P>0.9$, mixed when $0.1 \leqslant P \leqslant 0.9$, and Higgsino-like when $P<0.1$.

The unification conditions represent a theoretically attractive possibility, which makes strictly universal SUGRA models very predictive. However, the above assumptions, particularly (ii) and (iii), are not fully justified, since universality may occur at a scale higher than $M_{\mathrm{GUT}}$, i.e. the Planck scale or string scale, in which case renormalization above $M_{\mathrm{GUT}}$ weakens universality in the $m_{i}$. Deviations from some of the unification conditions have been considered by a number of authors [9-11]. Implications of these deviations for relic neutralino phenomenology have been discussed in detail in Refs. [9,11].

In the present paper, we discuss the DAMA/NaI data both in a SUGRA model with strict unification conditions and in a SUGRA framework, where we introduce a departure from universality in the scalar masses at $M_{\mathrm{GUT}}$ which splits the Higgs mass parameters $M_{H_{1}}$ and $M_{H_{2}}$ in the following way:

$$
M_{H_{i}}^{2}\left(M_{\mathrm{GUT}}\right)=m_{0}^{2}\left(1+\delta_{i}\right) .
$$

The parameters $\delta_{i}$ which quantify the departure from universality for the $M_{H_{i}}^{2}$ will be varied in the range $(-1,+1)$, but are taken to be independent of the other supersymmetric parameters.

Our supersymmetric parameter space is constrained by the following conditions: (a) all experimental bounds on Higgs boson, neutralino, chargino and sfermion masses are satisfied (for current CERN $e^{+} e^{-}$collider LEP bounds see, for instance, Refs. [12,13]), (b) the neutralino is the lightest supersymmetric particle (LSP), (c) the constraints on the $b$ $\rightarrow s+\gamma$ process are satisfied, (d) the constraints on the mass of the bottom quark $m_{b}$ are also satisfied, however, with a $b-\tau$ Yukawa unification relaxed by about $20 \%$, (e) EWSB is realized radiatively, (f) the neutralino relic abundance $\Omega_{\chi} h^{2}$ does not exceed the cosmological upper bound, which is conservatively set here as $\Omega_{\chi} h^{2} \leqslant 0.7$. Because of the requirements of radiative EWSB and of the universality conditions on the gaugino masses and on the trilinear couplings, the independent supersymmetric parameters are reduced to the following set (apart from the $\delta_{i}$ 's): $m_{1 / 2}, m_{0}, A_{0}, \tan \beta$ $=v_{2} / v_{1}$, and $\operatorname{sgn}(\mu)$.

The renormalization group equations (RGE's) are solved by using the one-loop beta functions including the whole supersymmetric particle spectrum from the GUT scale down to $M_{Z}$, neglecting the possible effects of intermediate thresholds. Two-loop and threshold effects on the running of the gauge and Yukawa couplings are known not to exceed $10 \%$ of the final result [14]. While this is of crucial impor- tance as far as gauge coupling unification is concerned [14], it is a second-order effect on the evolution of the soft masses and then it is neglected here.

In order to specify the supersymmetry phenomenology, boundary conditions for the gauge and Yukawa couplings have to be specified. Low-scale values for the gauge couplings and for the top-quark and the tau-lepton Yukawa couplings are fixed using present experimental results. In particular, we assign for the top mass the value $m_{t}=175 \mathrm{GeV}$.

A few qualifications are in order here about the constraints due to the $b \rightarrow s+\gamma$ process and for the bottom mass. In our analysis, the inclusive decay rate $\mathrm{BR}\left(B \rightarrow X_{s} \gamma\right)[15-$ $20]$ is calculated with corrections up to the leading order. Next-to-leading order corrections [21-24] are included only when they can be applied in a consistent way, i.e., both to standard model and to susy diagrams. This criterion limits the use of next-to-leading order corrections to peculiar regions of the supersymmetric parameter space, where the assumptions, under which the next-to-leading order susy corrections have been obtained, apply [24]. We require that our theoretical evaluation for $\operatorname{BR}\left(B \rightarrow X_{s} \gamma\right)$ is within the range $1.96 \times 10^{-4} \leqslant \mathrm{BR}\left(B \rightarrow X_{s} \gamma\right) \leqslant 4.32 \times 10^{-4}$. This range is obtained by combining the experimental data of Refs. [25,26] at $95 \%$ C.L. and by adding a theoretical uncertainty of $25 \%$, whenever the still incomplete next-to-leading order susy corrections cannot be applied.

The supersymmetric corrections to the bottom mass include contributions from bottom-squark-gluino loops and from top-squark-chargino loops [27]. In the present analysis, the bottom mass is computed as a function of the other parameters and required to be compatible with the present experimental bounds. Theoretical uncertainties in the evaluation of $m_{b}$ arise from the running of the RGE's. Since our choice is to solve RGE's at the one-loop level and without thresholds, we estimate an uncertainty of the order of $10 \%$ in our prediction for $m_{b}$. To take into account such an uncertainty we have chosen to weaken the bounds on $m_{b}$ given in Ref. [8] by $10 \%$. Thus we require $m_{b}$ to fall into the range $2.46 \mathrm{GeV} \leqslant m_{b}\left(M_{Z}\right) \leqslant 3.42 \mathrm{GeV}$, at $95 \%$ C.L. As mentioned above, $b-\tau$ Yukawa unification is relaxed by about $20 \%$.

The neutralino relic abundance $\Omega_{\chi} h^{2}$ is calculated as illustrated in Ref. [28]. As already stated, we apply to our supersymmetric parameter space an upper bound conservatively set at the value $\Omega_{\chi} h^{2} \leqslant 0.7$, and we consider as cosmologically interesting the range $0.01 \leqslant \Omega_{\chi} h^{2} \leqslant 0.7$. However, we stress that, according to the most recent data and analyses [29], the most appealing interval for the neutralino relic abundance is the narrower one: $0.02 \leq \Omega_{\chi} h^{2} \leqq 0.2$. The local neutralino density $\rho_{\chi}$ is factorized in terms of the total local dark matter density $\rho_{l}$ as $\rho_{\chi}=\xi \rho_{l}$. The parameter $\xi$ is calculated according to the usual rescaling recipe [30]: $\xi$ $=\min \left[\Omega_{\chi} h^{2} /\left(\Omega h^{2}\right)_{\min }, 1\right]$. We take here $\left(\Omega h^{2}\right)_{\min }=0.01$.

In our analysis the $m_{1 / 2}, m_{0}, A_{0}, \tan \beta$ parameters are varied in the following ranges: $10 \mathrm{GeV} \leqslant m_{1 / 2} \leqslant 500 \mathrm{GeV}$, $m_{0} \leqslant 1 \mathrm{TeV},-3 \leqslant A_{0} \leqslant+3,1 \leqslant \tan \beta \leqslant 50$; the parameter $\mu$ is taken positive. We remark that the values taken here as upper limits of the ranges for $m_{1 / 2}, m_{0}$ are inspired by the upper bounds which may be derived for these quantities in SUGRA theories, when one requires that the EWSB, radia- 
tively induced by the soft supersymmetry breaking, does not occur with excessive fine-tuning (see Ref. [9], and references quoted therein).

\section{RATE FOR WIMP DIRECT DETECTION}

The indication of a possible annual modulation effect, singled out by the DAMA/NaI data [1], points, at a $2 \sigma$ C.L., to a very delimited region in the plane $\xi \sigma_{\text {scalar }}^{\text {(nuclen }}-m_{\chi}$, where $m_{\chi}$ is the WIMP mass and $\sigma_{\text {scalar }}^{\text {(nucleon) }}$ is the WIMP-nucleon elastic scalar cross section. When the uncertainties in $\rho_{l}$ are taken into account, the original $2 \sigma$ C.L. region singled out by DAMA/NaI data has to be enlarged into a region $R$, where the quantity $\xi \sigma_{\text {scalar }}^{\text {(nucleon) }}$ falls into the following range [3]: $(1-3) \times 10^{-9} \mathrm{nb} \leq \xi \sigma_{\text {scalar }}^{\text {(nucleon })} \leqslant 3 \times 10^{-9} \mathrm{nb}$, in the mass range $30 \mathrm{GeV} \leq m_{\chi} \leq 110 \mathrm{GeV}$.

In Ref. [3], where the relevant formulas for the evaluation of $\sigma_{\text {scalar }}^{\text {(nucleon }}$ are reported, it is also shown that, in the MSSM, wide domains of the supersymmetric parameter space provide values for $\sigma_{\text {scalar }}^{\text {(nucleon) }}$ and $m_{\chi}$ which are within region $R$. Out of the two competing contributions to $\sigma_{\text {scalar }}^{\text {(nucleon) }}$, Higgsexchange and squark-exchange processes, usually the former dominates over the latter one. Then let us discuss which properties of the Higgs-exchange amplitude are instrumental in making this contribution sizeable enough as required by the modulation effect $\xi \sigma_{\text {scalar }}^{\text {(nuclen })} \geq 1 \times 10^{-9} \mathrm{nb}$. As is clear from the expressions given in Ref. [3], the most important parameters for establishing the size of the Higgs-exchange amplitude are $m_{h}, \tan \beta$, and the mixing angle $\alpha$ of the two $C P$-even neutral Higgs bosons $(h$ and $H$ ). The largest values for the Higgs-exchange amplitude occur for the following combination of their respective values: small $m_{h}$ (because of the propagator), large values of $\tan \beta$, and of $\alpha$ (because of the structure of the Higgs-quark couplings). In SUGRA these three parameters are rather strongly correlated [9], so that requiring some lower bound $\xi \sigma_{\text {scalar }}^{\text {(nucleon) imposes }}$ severe constraints on the parameter space. These properties may be suitably discussed in terms of the mass $m_{A}$ of the $C P$-odd neutral Higgs boson (however, we remind the reader that this parameter is not free, but depends on the parameters defining our parameter space and on the RGE's evolution).

From Fig. 1, which displays a generic scatter plot of $\xi \sigma_{\text {scalar }}^{\text {(nucleon }}$ versus $m_{A}$ in the universal SUGRA model (i.e., with $\delta_{i}$ 's $=0$ ), we see that the lower bound $\xi \sigma_{\text {scalar }}^{\text {(nuclen) }} \gtrless$ $1 \times 10^{-9} \mathrm{nb}$ implies the upper bound $m_{A} \lesssim 180 \mathrm{GeV}$. If we further take into account the scatter plot of $m_{A}$ in terms of $\tan \beta$, shown in Fig. 2, we obtain in turn a lower limit on $\tan \beta: \tan \beta \gtrsim 42$. We also note that just a small relaxation in the lower bound for $\xi \sigma_{\text {scalar }}^{\text {(nucleon) }} \geq 1 \times 10^{-9} \mathrm{nb}$ would also allow intermediate values of $\tan \beta: \tan \beta \sim 5-10$. The reasons for the typical feature of the scatter plot of Fig. 2 for $m_{A}$ $\$ 300 \mathrm{GeV}$ are discussed in the Appendix.

If we now require our supersymmetric configurations to lie inside the modulation region $R$ (this set of configurations is denoted as set $S$ ), we obtain the scatter plot of Fig. 3, which proves that the annual modulation data are in fact compatible with a universal SUGRA scheme. Figure 4 shows that a number of the selected supersymmetric configurations

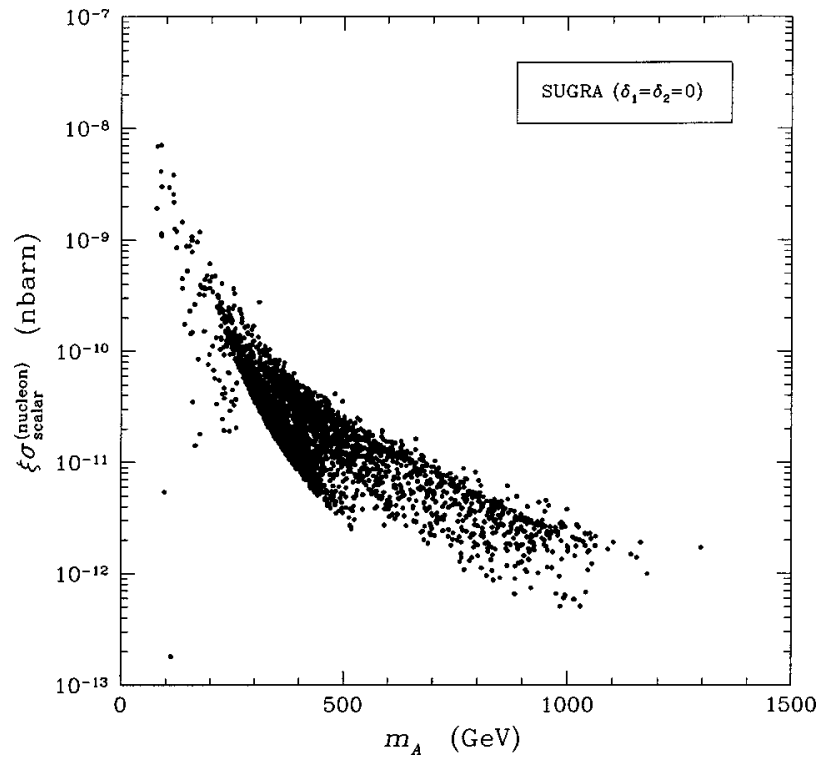

FIG. 1. Scatter plot of $\xi \sigma_{\text {scalar }}^{\text {(nucleon) }}$ versus $m_{A}$ for a scanning of the supersymmetric parameter space as defined in Sec. II, in universal SUGRA.

fall into the cosmological interesting range of $\Omega_{\chi} h^{2}$.

The other qualifications for the configurations which lie inside the region $R$, relevant for searches at accelerators, concern the ranges for the $h$-Higgs boson mass, the neutralino mass and the lightest top-squark mass, which we find to be $m_{h} \lesssim 115 \mathrm{GeV}, 50 \mathrm{GeV} \lesssim m_{\chi} \lesssim 100 \mathrm{GeV}$, and $200 \mathrm{GeV} \lesssim m_{\tilde{t}_{1}} \leq 700 \mathrm{GeV}$, respectively.

Let us now turn to SUGRA schemes with deviations from universal scalar masses (i.e., $\delta_{i}$ 's $\neq 0$ ). By varying the usual supersymmetric parameters as before and the $\delta_{i}$ 's in the range $-1 \leqslant \delta_{i} \leqslant+1$ we find the scatter plot of Fig. 5, which shows that the requirement $\xi \sigma_{\text {scalar }}^{(\text {(nucleon })} \geq 1 \times 10^{-9}$ nb implies

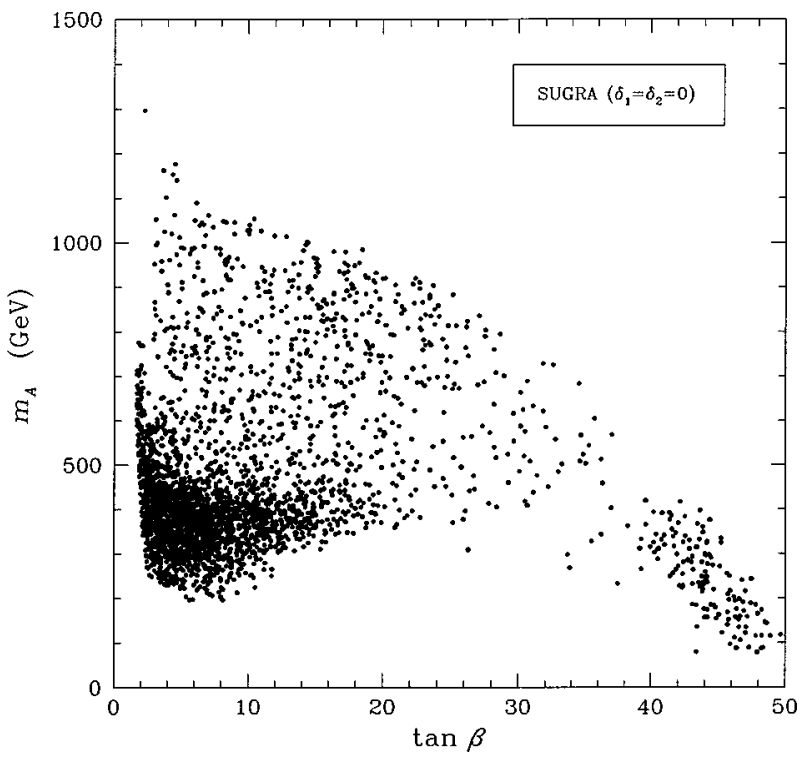

FIG. 2. Scatter plot of $m_{A}$ versus $\tan \beta$ for a scanning of the supersymmetric parameter space as defined in Sec. II, in universal SUGRA. 


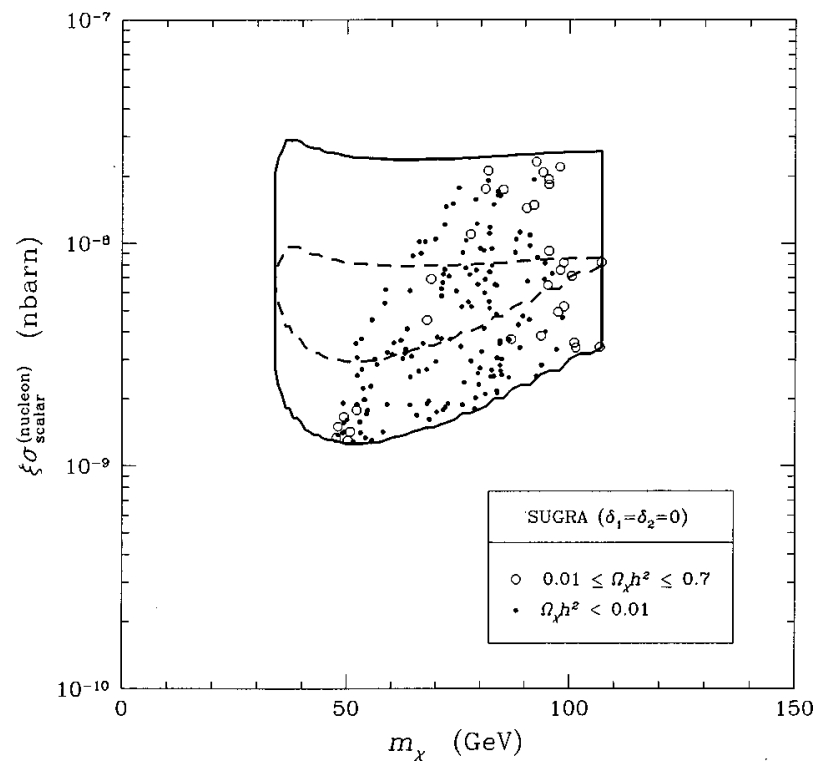

FIG. 3. Scatter plot of set $S$ in the plane $m_{\chi^{-}} \xi \sigma_{\text {scalar }}^{\text {(nucleon })}$ in universal SUGRA. The dashed contour line delimits the 2- $\sigma$ C.L. region, obtained by the DAMA/NaI Collaboration, by combining together the data of the two running periods of the annual modulation experiment [1]. The solid contour line is obtained from the dashed line, which refers to the value $\rho_{l}=0.3 \mathrm{GeV} \mathrm{cm}^{-3}$, by accounting for the uncertainty range of $\rho_{l}$, as explained in Sec. III (the region delimited by the solid line is denoted as region $R$ in the text). The representative points are denoted differently depending on the values of the neutralino relic abundance $\Omega_{\chi} h^{2}$.

now a more relaxed upper bound on $m_{A}: m_{A} \lesssim 330 \mathrm{GeV}$. From Fig. 6, we see that this upper limit on $m_{A}$ is compatible with all values of $\tan \beta$. In Figs. 7 and 8 we notice that, as expected, our new sample of representative points covers a

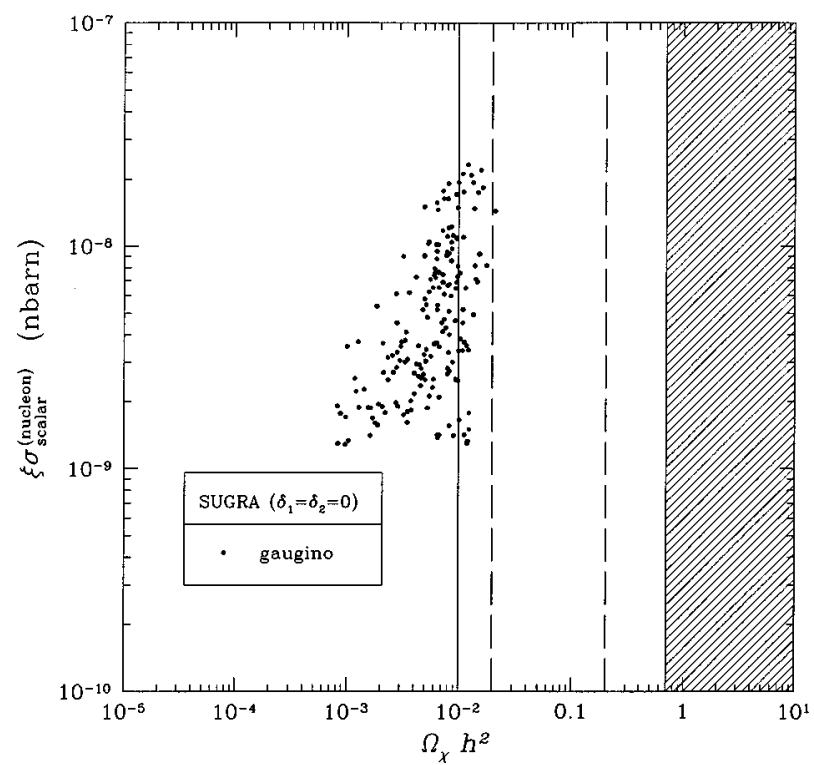

FIG. 4. Scatter plot of set $S$ in the plane $\Omega_{\chi} h^{2}-\xi \sigma_{\text {scalar }}^{\text {(nucleon) }}$ in universal SUGRA. Here neutralinos turn out to be gaugino-like only. The two vertical solid lines delimit the $\Omega_{\chi} h^{2}$ range of cosmological interest. The two dashed lines delimit the most appealing interval for $\Omega_{\chi} h^{2}$, as suggested by the most recent observational data.

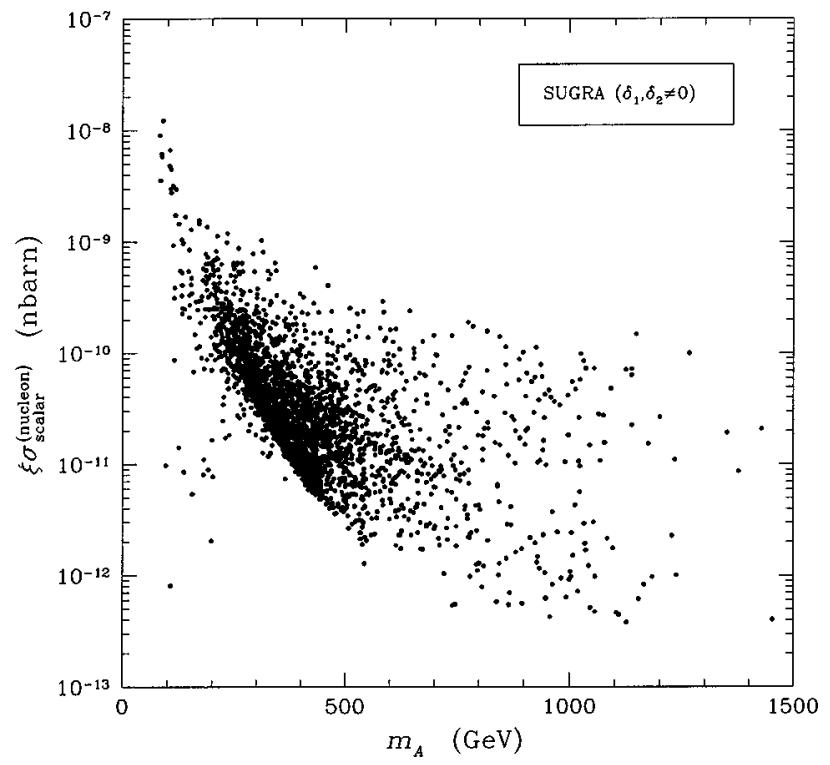

FIG. 5. Scatter plot of $\xi \sigma_{\text {scalar }}^{\text {(nucleon) }}$ versus $m_{A}$ for a scanning of the supersymmetric parameter space as defined in Sec. II, in nonuniversal SUGRA.

slightly wider domain of region $R$, and, more significantly, contains new neutralino configurations of cosmological interest. Figures $9-11$ show how the values for the $h$-Higgs boson mass, the neutralino mass and the lightest top-squark mass are distributed in terms of $\tan \beta$. The ranges of these masses are similar to those already found in the universal case, but now $\tan \beta$ extends to the interval $10 \leqq \tan \beta \leq 50$, instead of being limited only to very large values.

The distribution of the values for the parameters $\delta_{i}$ 's, which provide supersymmetric configurations in agreement with the annual modulation data, are shown in Fig. 12. The peculiar distribution of representative points in the left-upper

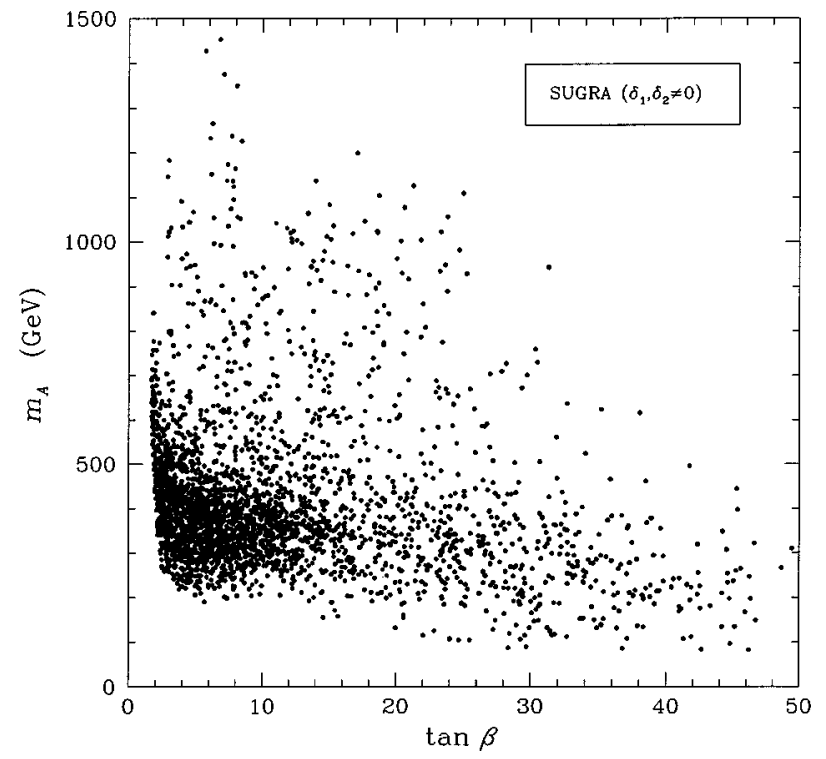

FIG. 6. Scatter plot of $m_{A}$ versus $\tan \beta$ for a scanning of the supersymmetric parameter space as defined in Sec. II, in nonuniversal SUGRA. 


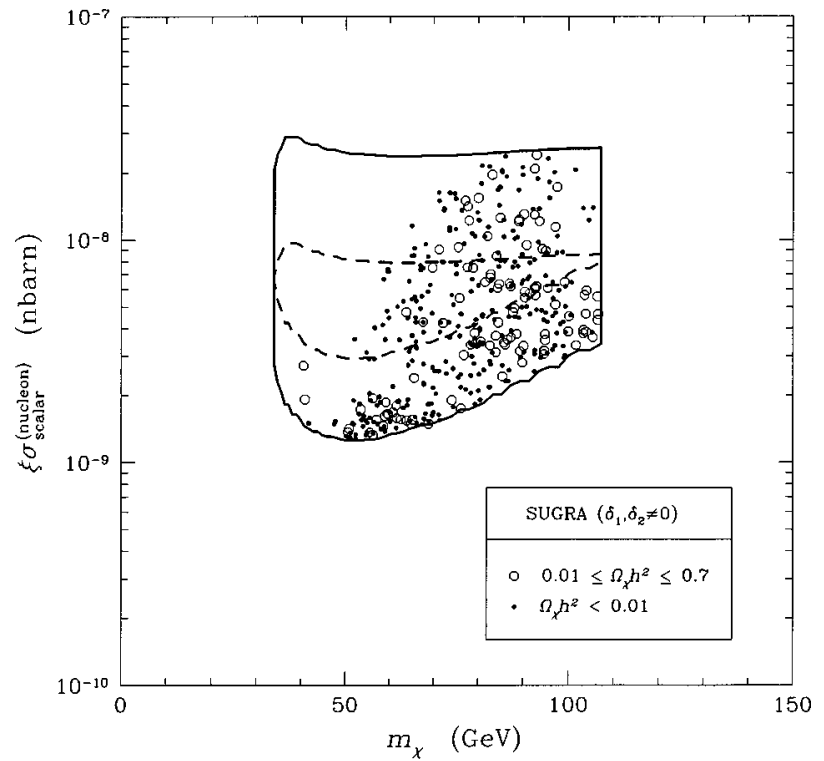

FIG. 7. As in Fig. 3, for nonuniversal SUGRA.

side of the figure may be easily understood in terms of the general properties discussed in Ref. [9]. The generic trend displayed in this figure shows that small values of $\tan \beta$ require sizeable deviations from universality in the Higgs boson mass parameters.

\section{CONCLUSIONS}

We have analyzed the total sample of new and former DAMA/NaI data [1,2], which provide the indication of a possible annual modulation effect in the rate for WIMP direct detection. We have demonstrated that these experimental data, already proved to be widely compatible with relic neutralinos of cosmological interest in a MSSM scheme

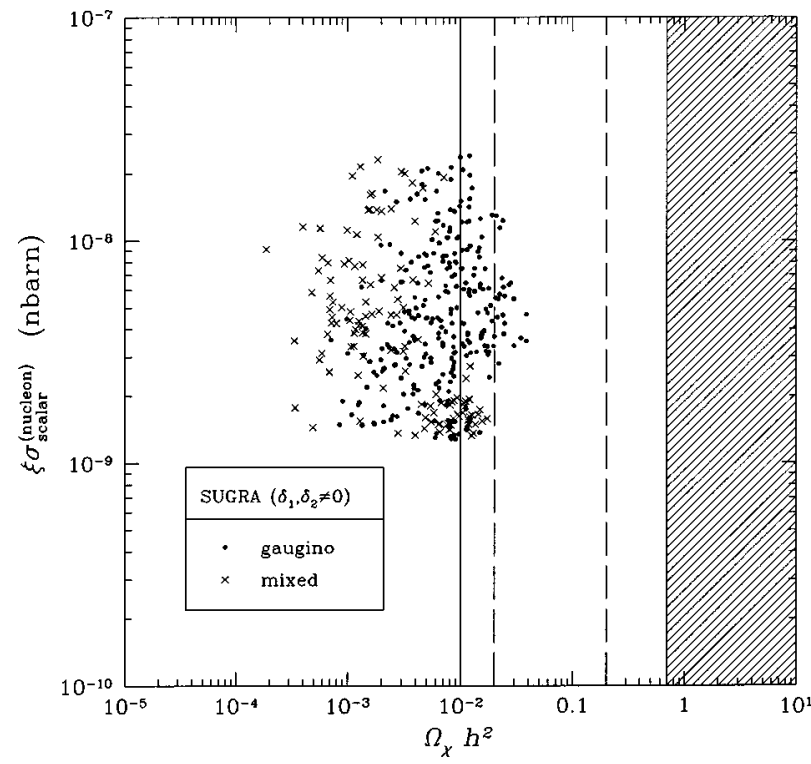

FIG. 8. As in Fig. 4, for nonuniversal SUGRA. Here dots and crosses denote neutralinos of different composition according to the classification in Sec. II.

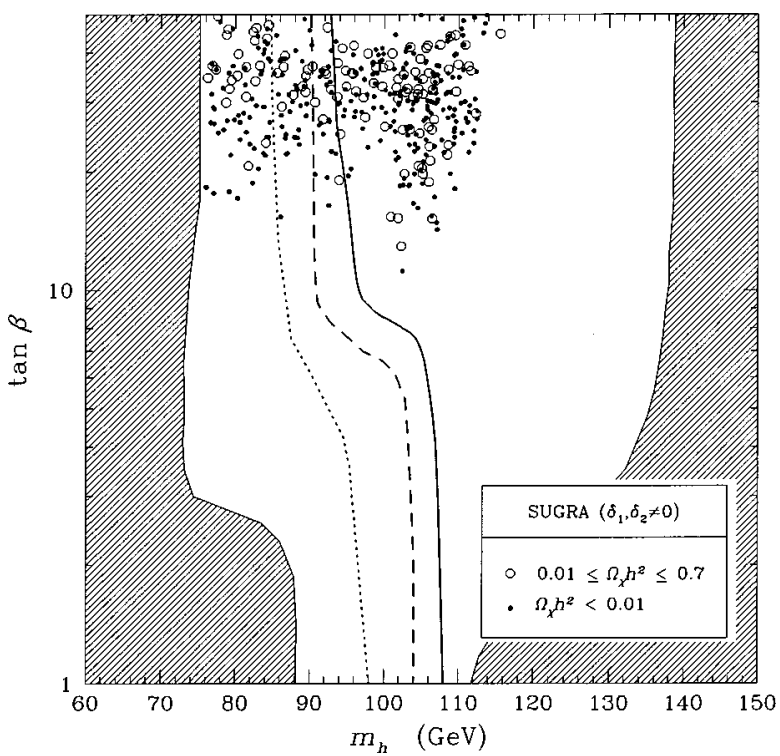

FIG. 9. Scatter plot of set $S$ in the plane $m_{h}-\tan \beta$, in nonuniversal SUGRA. The hatched region on the right is excluded by theory. The hatched region on the left is excluded by present LEP data at $\sqrt{s}=183 \mathrm{GeV}$. The dotted and the dashed curves denote the reach of LEP2 at energies $\sqrt{s}=192 \mathrm{GeV}$ and $\sqrt{s}=200 \mathrm{GeV}$, respectively. The solid line represents the $95 \%$ C.L. bound reachable at LEP2, in the case of nondiscovery of a neutral Higgs boson.

$[3,5]$, are also compatible with a SUGRA framework.

We have specifically considered a supergravity scheme with strict unification conditions on scalar and gaugino masses and on trilinear scalar couplings, and supergravity models with departures from universality in the scalar mass parameters of the Higgs sector. We have proved that in universal SUGRA neutralino configurations with interesting

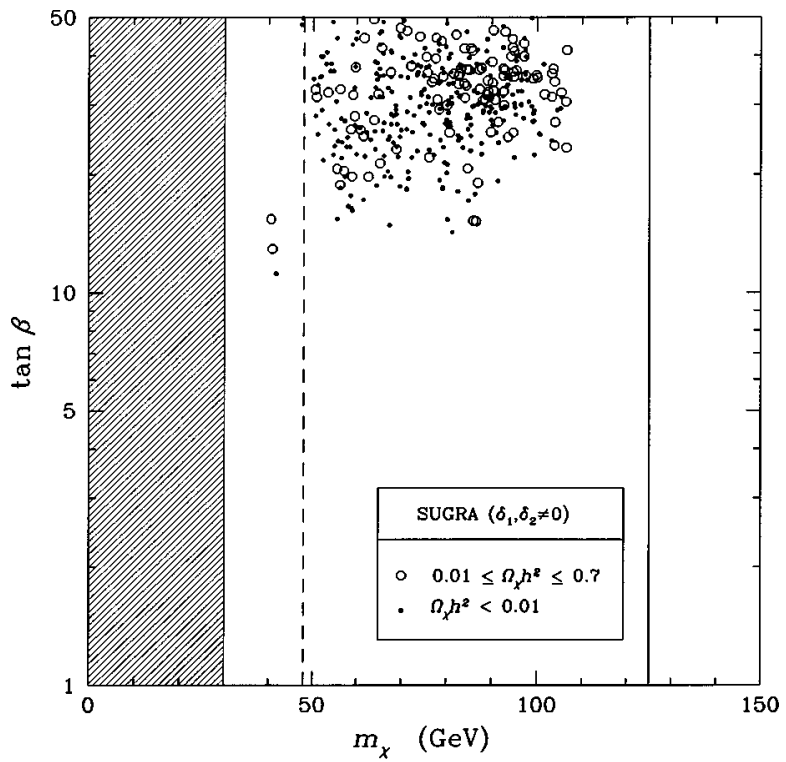

FIG. 10. Scatter plot of set $S$ in the plane $m_{\chi}$-tan $\beta$, in nonuniversal SUGRA. The hatched region on the left is excluded by present LEP data. The dashed and the solid vertical lines denote the reach of LEP2 and TeV33, respectively. 


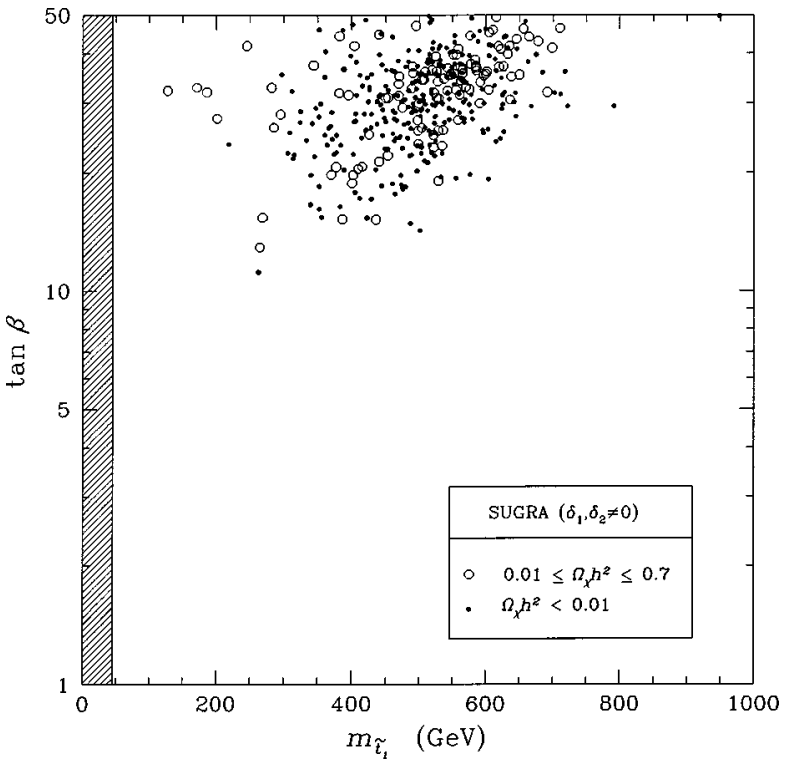

FIG. 11. Scatter plot of set $S$ in the plane $m_{\tilde{t}_{1}}-\tan \beta$, in nonuniversal SUGRA. The hatched region is excluded by LEP data (without any restriction on other masses).

cosmological properties may be found. Even more so, in case of non-universal SUGRA models.

Other main results of our analysis are the following. In the universal SUGRA model the constraints imposed by the DAMA NaI data imply for the $h$-Higgs boson mass, the neutralino mass, and the lightest top-squark mass, the following ranges: $m_{h} \lesssim 115 \mathrm{GeV}, 50 \mathrm{GeV} \lesssim m_{\chi} \lesssim 100 \mathrm{GeV}$, and 200 $\mathrm{GeV} \lesssim m_{\tilde{t}_{1}} \leq 700 \mathrm{GeV}$, respectively. In universal SUGRA $\tan \beta$ is constrained to be large, $\tan \beta \gtrsim 42$, whereas, with departure from universality in the scalar masses, the range for $\tan \beta$ widens to $10 \leqq \tan \beta \leqq 50$.

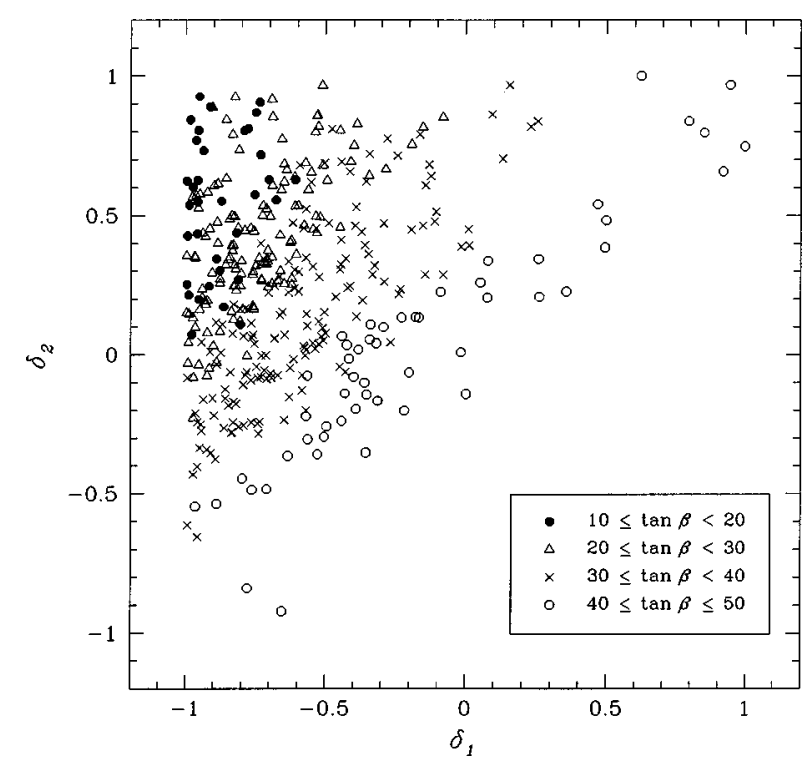

FIG. 12. Scatter plot of the values of the $\delta_{1}$ and $\delta_{2}$ parameters for set $S$.

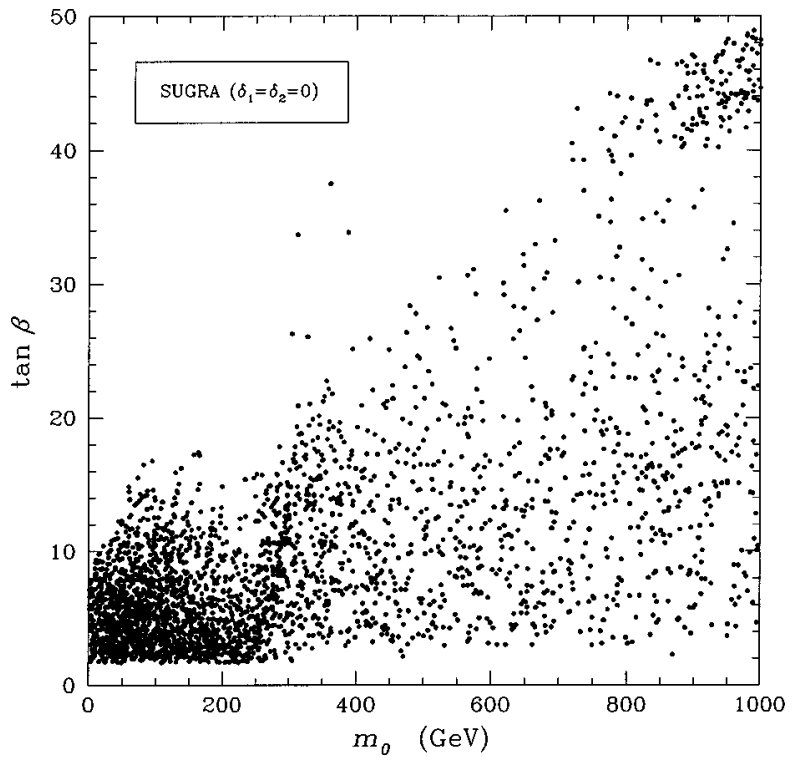

FIG. 13. Scatter plot in the plane $m_{0}$-tan $\beta$, for a scanning of the supersymmetric parameter space as defined in Sec. II, in universal SUGRA.

\section{APPENDIX}

In this appendix we show that the peculiar behavior of the scatter plot in Fig. 2 for $m_{A} \lesssim 300 \mathrm{GeV}$ is induced, in the universal SUGRA model, by the combined effect of the limits on the $b \rightarrow s+\gamma$ decay rate and on the mass of the bottom quark $m_{b}$. Let us start by reminding that in generic SUGRA models $m_{A}$ is a function of the other parameters through the radiatively induced EWSB mechanism. A useful parametrization is [9]

$$
m_{A}^{2}=K_{1} m_{1 / 2}^{2}+K_{2} m_{0}^{2}+K_{3} A_{0}^{2} m_{0}^{2}+K_{4} A_{0} m_{0} m_{1 / 2}-m_{Z}^{2},
$$

where the coefficients $K_{i}$ are only functions of $\tan \beta$ and the $\delta_{i}$ 's. Their properties are extensively discussed in Ref. [9], to which we refer for details. To simplify the discussion let us take $A_{0}=0$.

In the universal SUGRA model the coefficients $K_{1}$ and $K_{2}$ are both decreasing functions of $\tan \beta$, being $K_{1} \sim 3$ and $K_{2} \sim 1$ for $\tan \beta \sim 5-10$ and both vanishingly small at large $\tan \beta$. Actually, while $K_{1}$ is always positive, $K_{2}$ becomes negative for $\tan \beta \sim 50$. Furthermore $m_{1 / 2}$ is limited from above by the annual modulation data which imply $m_{\chi}$ $\lesssim 110 \mathrm{GeV}$ and then $m_{1 / 2} \sim 2.5 m_{\chi} \lesssim 270 \mathrm{GeV}$ (the relation $m_{1 / 2} \sim 2.5 m_{\chi}$ holds, since neutralino is mainly gaugino-like in universal SUGRA). Instead, for $m_{0}$ we have a lower bound which depends on $\tan \beta$, as displayed in Fig. 13. This bound arises as a combined effect of the $b \rightarrow s+\gamma$ and $m_{b}$ constraints. Consequently $m_{A}$ may be small only for $\tan \beta$ $\lesssim 10$, where $m_{0}$ may be arbitrarily small, or at large $\tan \beta$, where the product $K_{2} m_{0}^{2}$ is kept small by $K_{2}$. In SUGRA models with $\delta_{i} \neq 0$ the coefficient $K_{2}$ may become vanishingly small also for intermediate values of $\tan \beta$ so that, in non-universal models, small values of $m_{A}$ may occur over the whole $\tan \beta$ range, as shown in Fig. 6. 
[1] R. Bernabei et al., Phys. Lett. B (to be published), Roma II University Report Nos. ROMA2F/98/27; Riv. Nuovo Cimento (to be published), ROMA2F/98/34, 1998.

[2] R. Bernabei et al., Phys. Lett. B 424, 195 (1998).

[3] A. Bottino, F. Donato, N. Fornengo and S. Scopel, preceding paper, Phys. Rev. D 59, 095003 (1999).

[4] H. P. Nilles, Phys. Rep. 110, 1 (1984); H. E. Haber and G. L. Kane, ibid. 117, 75 (1985); R. Barbieri, Riv. Nuovo Cimento 11, 1 (1988).

[5] A. Bottino, F. Donato, N. Fornengo, and S. Scopel, Phys. Lett. B 423, 109 (1998).

[6] An analysis of the former modulation data (collected by the DAMA/NaI experiment in the running period No. 1) in terms of SUGRA models has been presented by R. Arnowitt, in International Conference on High Energy Physics (ICHEP98), Vancouver, 1998 (unpublished).

[7] L. E. Ibañez and C. Lopez, Nucl. Phys. B233, 511 (1984); J. Ellis and F. Zwirner, ibid. B338, 317 (1990); M. Drees and M. M. Nojiri, ibid. B369, 54 (1992); R. Arnowitt and P. Nath, Texas A\&M University Report No. CTP-TAMU-52-93, hep-ph/9309277, 1993; J. L. Lopez, D. V. Nanopoulos, and A. Zichichi, Riv. Nuovo Cimento 17, 1 (1994); W. de Boer, Prog. Part. Nucl. Phys. 33, 201 (1994); G. L. Kane, C. Kolda, L. Roszkowski, and J. D. Wells, Phys. Rev. D 49, 6173 (1994); R. Rattazzi and U. Sarid, ibid. 53, 1553 (1996); X. Wang, J. Lopez, and D. Nanopoulos, Phys. Lett. B 348, 105 (1995); V. Barger, M. S. Berger, and P. Ohmann, Phys. Rev. D 49, 4908 (1994).

[8] P. H. Chankowski, J. Ellis, M. Olechowski, and S. Pokorski, CERN Report No. CERN-TH/98-119, hep-ph/9808275, 1998.

[9] V. Berezinsky, A. Bottino, J. Ellis, N. Fornengo, G. Mignola, and S. Scopel, Astropart. Phys. 5, 1 (1996).

[10] N. Polonsky and A. Pomarol, Phys. Rev. Lett. 73, 2292 (1994); Phys. Rev. D 51, 6532 (1995); M. Olechowski and S. Pokorski, Phys. Lett. B 344, 201 (1995); D. Matalliotakis and H. P. Nilles, Nucl. Phys. B435, 115 (1995); A. Pomarol and S. Dimopoulos, ibid. B453, 83 (1995); H. Murayama, Berkeley Report No. LBL-36962, 1995; P. Nath and R. Arnowitt, Phys. Rev. D 56, 2820 (1997).

[11] V. Berezinsky, A. Bottino, J. Ellis, N. Fornengo, G. Mignola, and S. Scopel, Astropart. Phys. 5, 333 (1996).

[12] M. Felcini, in Workshop "From Planck Scale to Electroweak
Scale,' Kazimierz, 1998; A. Lipniacka, ibid; ALEPH Collaboration, R. Barate et al., Phys. Lett. B 433, 176 (1998); L3 Susy Group, L3 Note 2238, 1998 (unpublished).

[13] K. Desch and P. Rebecchi, talks at International Conference on High Energy Physics, Vancouver, 1998 (unpublished).

[14] P. Langacker and N. Polonsky, Phys. Rev. D 47, 4028 (1993).

[15] S. Bertolini, F. Borzumati, A. Masiero, and G. Ridolfi, Nucl. Phys. B353, 591 (1991).

[16] R. Barbieri and G. F. Giudice, Phys. Lett. B 309, 86 (1993).

[17] R. Garisto and J. N. Ng, Phys. Lett. B 315, 372 (1993).

[18] F. M. Borzumati, M. Drees, and M. M. Nojiri, Phys. Rev. D 51, 341 (1995).

[19] J. Wu, R. Arnowitt, and P. Nath, Phys. Rev. D 51, 1371 (1995).

[20] V. Barger, M. S. Berger, P. Ohmann, and R. J. N. Phillips, Phys. Rev. D 51, 2438 (1995).

[21] K. Chetyrkin, M. Misiak, and M. Münz, Phys. Lett. B 400, 206 (1997).

[22] M. Ciuchini, G. Degrassi, P. Gambino, and G. F. Giudice, Nucl. Phys. B527, 21 (1998).

[23] A. Czarnecki and W. J. Marciano, Phys. Rev. Lett. 81, 277 (1998).

[24] M. Ciuchini, G. Degrassi, P. Gambino, and G. F. Giudice, Nucl. Phys. B534, 3 (1998).

[25] CLEO Collaboration, S. Glenn, Report No. CLEO CONF 9817, 1998; in International Conference on High Energy Physics, Vancouver, 1998, paper No. 1011 (unpublished).

[26] ALEPH Collaboration, R. Barate et al., Phys. Lett. B 429, 169 (1998).

[27] M. Carena, M. Olechowski, S. Pokorski, and C. E. M. Wagner, Nucl. Phys. B426, 269 (1994); R. Rattazzi and U. Sarid, Phys. Rev. D 50, 7048 (1994).

[28] A. Bottino, V. de Alfaro, N. Fornengo, G. Mignola, and M. Pignone, Astropart. Phys. 2, 67 (1994).

[29] W. L. Freedman, R. Kirshner, and C. Lineweaver, talks given at International Conference of Cosmology and Particle Physics, Geneva, 1998, http://wwwth.cern.ch/capp98/ programme.html; M. White, Astrophys. J. 506, 495 (1998); N. A. Bahcall and X. Fan, astro-ph/9804082; C. Lineweaver, astro-ph/9805326.

[30] T. K. Gaisser, G. Steigman, and S. Tilav, Phys. Rev. D 34, 2206 (1986). 